\title{
Inference of Planck action constant by a classical fluctuative postulate holding for stable microscopic and macroscopic dynamical systems
}

\author{
Salvatore De Martino ${ }^{a, b, *}$, Silvio De Siena ${ }^{a, b, \dagger}$ and Fabrizio Illuminati ${ }^{a, b, c, \ddagger}$ \\ ${ }^{a}$ Dipartimento di Fisica, \\ ${ }^{b}$ INFN, Sez. di Napoli and INFM Unità di Salerno, \\ Università di Salerno, I-84081 Baronissi (SA) Italy, \\ ${ }^{c}$ Fakultät für Physik, Universität Potsdam, \\ Am Neuen Palais 19, D-14469, Potsdam, Germany.
}

(4 August 1998)

\begin{abstract}
The possibility is discussed of inferring or simulating some aspects of quantum dynamics by adding classical statistical fluctuations to classical mechanics. We introduce a general principle of mechanical stability and derive a necessary condition for classical chaotic fluctuations to affect confined dynamical systems, on all scales ranging from microscopic to macroscopic domains. As a consequence we obtain, both for microscopic and macroscopic aggregates, dimensional relations defining the minimum unit of action of individual constituents, yielding in all cases Planck action constant.
\end{abstract}

PACS Numbers: 03.65.Bz; 05.45.+b; 05.40.+j

\section{INTRODUCTION}

Since the origin of quantum mechanics, the question of its relationship to classical mechanics has been recognized to be crucial for a deeper physical understanding of the theory.

The WKBJ analysis of the semiclassical limit and the coherent states of the harmonic oscillator are classic examples that show all the subtleties, both mathematical and conceptual, of the classic to quantum interplay.

The issue has recently raised widespread interest on account of remarkable experimental advances in the study of mesoscopic and macroscopic quantum phenomena such as high $T_{c}$ superconductivity, atom cooling and Bose-Einstein condensation, and the coherent quantum-like states of charged beams in particle accelerators. At the same time, for some theoretical problems such as quantum computing and the quantization of gravity, semiclassical methods have so far been the main or sometimes the only tool that led to some partial results.

Traditionally, the main theoretical tools employed to recover classical aspects of quantum systems and study the transition between the classical and the quantum domain fall in two classes: on the one hand asymptotic series expansions in powers of $\hbar$, such as the semiclassical WKBJ approximation; on the other hand, the singling out of particular quantum states exhibiting some degree of space and time coherent behavior.

In all such cases the arrow points in the direction going from the deep quantum domain heading on to recover classical aspects of the dynamics in some suitable limit.

In the present paper we show, in a still semi-quantitative framework, that it is possible to take the opposite route, namely to infer fundamental quantum aspects by starting from classical mechanics and then adding suitable, purely classical fluctuations to the classical deterministic motion: these fluctuations then simulate or describe some features of the fundamental quantum noise.

*E-Mail: demartino@vaxsa.csied.unisa.it

${ }^{\dagger} \mathrm{E}-$ Mail: desiena@vaxsa.csied.unisa.it

${ }^{\ddagger}$ E-Mail: illuminati@vaxsa.csied.unisa.it 
Remarkably, one needs to assume only a minimum set of very general properties about the fluctuations, the analysis of their detailed structure being, at this stage, unnecessary. In particular, they are assumed to be universal (i.e. independent of the systems and of the interactions considered), and to act coherently on all length scales ("coherent tremor hypothesis"). The scale of action of these fluctuations then naturally turns out to be, in all cases considered, the Planck constant. Most remarkable is that this occurence happens to be true also for classical macroscopic systems whose dynamics is commonly described in the framework of classical mechanics. A preliminary announcement containing partial results appeared in [1].

The starting point for our analysis is a scheme recently proposed by Francesco Calogero [2]. In his reasoning Calogero suggests that the origin of quantization be attributed to the universal interaction of every particle with the background gravitational force due to all other constituents of the Universe, which should result in some effects of chaoticity and stochasticity on sufficiently small space-time scales.

Assuming such a noisy component of the motion to be a coherent tremor in the sense we have explained above, and assuming a basic granularity of the Universe, made up of nucleons of mass $m$ interacting via the gravitational force, Calogero derives an expression for Planck's constant, $h=G^{1 / 2} m^{3 / 2} R^{1 / 2}$, with $G$ the Newtonian gravitational constant and $R$ the observed radius of the Universe. This formula, which connects the fundamental constant of quantum theory with the fundamental gravitational constants, was already known since some time [3], but its meaning and implications were so far unexplained.

In the present paper we reformulate the scheme of Calogero dropping the hypothesis of the gravitational chaos, and replacing it with the assumption of a general principle of stability for classical dynamical systems. The requirement of mechanical stability holds independently of the interaction being considered (as long as the latter is attractive), and allows us to derive the coherent tremor hypothesi of Calogero, rather than postulating it.

The method can then be applied, besides gravity, to all other interactions, for instance electromagnetic and strong forces. In this way we are able to show that for any bound and stable aggregate of particles interacting through any (overall attractive) phenomenological law of force, the coherent tremor hypothesis yields precise dimensional relations between the minimum unit of action per particle, the fundamental constants associated to the given interaction, and the dimension (radius) of stability of the given aggregate.

In order of magnitude, such dimensional relations always yield Planck action constant $h$, and this is the main result of the present work.

The extension to finite temperature, and other dimensional estimates that come as applications of the method are also discussed in the paper, which is organized as follows.

After a brief review in Sect. 2 of the original model introduced by Calogero, we reformulate in Sect. 3 the problem in a general form combining the classical laws of force with a simple principle of dynamic stability. The coherent tremor hypothesis of Calogero is then readily derived in a very natural way.

We then show in Sect. 4 that the coherent tremor hypothesis can be reformulated in a "Keplerian" version which is independent of the number of particles (constituents) of the dynamical system under consideration. In the case of astrophysical systems associated to gravitational interactions, this form recovers already known features of some long-range classical cosmological scaling laws [3].

In Sect. 5 we apply the method to all possible interactions (gravitational, electromagnetic, strong) giving rise to stable bound systems of particles. We find that, in each of such cases, the order of magnitude of the characteristic unit of action per particle (constituent) equals that of Planck constant. Remarkably, we consider also the case of macroscopic, therefore substantially classical, aggregates of charged particles like neutral plasmas and, particularly, charged particle beams in accelerators. The method yields, even for these classical systems, a unit of action per constituent of the order of $h$. Estimates on the phenomenological ranges of weak, screened electromagnetic interactions for mesoscopic systems are then derived; for electrostatic dipolar forces, we obtain that the unit of action per particle is directly linked to the fine structure constant, and we comment on this intriguing connection.

In Sect. 6, a reasonable hypothesis on the form of the characteristic time scale of the classical fluctuations, already introduced by Calogero, combined with the natural definition of a thermodynamic 
equilibrium energy allows us to extend the procedure to finite temperature, and to check the consistency of our scheme in several interesting cases. In particular, we obtain a general expression for the equivalent thermal unit of action, that scales in a nontrivial way as teh square root of the number of constituents. Applyinf this definition to the instance of charged particle beams we link the beam unit of emittance to the number of particles in the beam. Among other quantities that we can estimate by our method, we have reported the characteristic velocity of a condensed particle in a Bose-Einstein condensate, and the order of magnitude of the total number of nucleons in the Universe, in excellent agreement with the estimates obtained by cosmological arguments.

Finally, in Sect. 7 we comment on the nature and meaning of our results, and draw our (tentative) conclusions, expecially by discussing about the possible future perspectives and directions of research along the line introduced in the present paper.

Before entering the detailed discussion, we warn the reader that throughout all computations we have neglected all numerical factors that do not affect the order of magnitude of the estimated quantities.

\section{THE SCHEME OF CALOGERO: THE COHERENT TREMOR HYPOTHESIS}

The fundamental assumption in the scheme of Calogero [2] is that, due to the universal and long-ranged nature of the gravitational interaction, to the granular structure of the Universe (made of an exceedingly large number of microscopic atomic constituents, for instance nucleons), and to the unavoidable presence of a chaotic component of the motion entailed by classical mechanics for a large many-body system, every and each particle in the Universe experiences locally the same stochastic gravitational acceleration. Calogero identifies in this phenomenon the universal stochastic background responsible for quantization.

Therefore, in his view, quantum mechanics should be derivable by classical mechanics plus a suitable ad hoc assumption on the nature and structure of the gravitational background stochastic fluctuations: the crucial point in the procedure is then the introduction of the characteristic time scale $\tau$ of the local, stochastic component of the motion for each single constituent.

Calogero assumes, rather naturally, that the characteristic frequency of the chaotic motion must be larger, the larger the number $N$ of elementary constituents in the Universe. Moreover, being this a collective stochastic effect, he infers that the fluctuations should be normally distributed and therefore scale proportionally to $\sqrt{N}$.

Hence, he puts forward the estimate

$$
\tau \cong N^{-1 / 2} \mathcal{T},
$$

where $\mathcal{T}$ is the characteristic scale of time for the mean global deterministic motion of each constituent in the Universe. Eq. (1) is the mathematical expression of the universal tremor hypothesis.

He defines also the characteristic global units of energy $E$ and of action $A$ of the Universe, and the unit of energy per particle (constituent) $\epsilon$ :

$$
\begin{aligned}
& A \cong E \mathcal{T}, \\
& \epsilon \cong E / N .
\end{aligned}
$$

Finally, he introduces the characteristic unit of action per particle $\alpha$, defined by

$$
\alpha \cong \epsilon \tau \text {. }
$$

The definitions (2), (3), (4) and the assumption (1) connect the unit of action $\alpha$ to the total action $A$ through the nontrivial relation

$$
\alpha \cong N^{-3 / 2} A .
$$


If $M$ is the total mass of the Universe, $R$ its observed radius and $G$ the universal gravitational constant, a simple dimensional analysis yields

$$
A \cong G^{1 / 2} M^{3 / 2} R^{1 / 2} .
$$

Estimating the total number of particles by $N \cong M / m$, with $m$ the mass of a nucleon (or of a hydrogen atom), Calogero finally obtains

$$
\alpha \cong G^{1 / 2} m^{3 / 2} R^{1 / 2} .
$$

Inserting the numerical values $m \cong 10^{-27} \mathrm{Kg}, G \cong 10^{-11} \mathrm{Kg}^{-1} \mathrm{~m}^{3} \mathrm{~s}^{-2}$, and the most updated cosmological estimate for the observed radius of the Universe $R \cong 10^{30} \mathrm{~m}$ [四, eq. (7) yields, in order of magnitude, $\alpha \cong h$, i.e. Planck action constant.

This estimate leads Calogero to infer the congruence and the cogency of his hypothesis, for classical gravity plus the tremor hypothesis (1) allows him to derive Planck constant. He provides also a semiquantitative argument (based on a simple nonrelativistic Newtonian model) to show that the above picture is consistent only with a spatially coherent character of the tremor, in the sense that the (tiny) corrections to the relative displacements between particles due to the stochastic background must be proportional to the relative displacements themselves, through a term which might depend on time. He refers to this large-scale coherence by the fascinating definition "breathing of the Universe" [2].

Concluding the section we wish to recall that the picture of Calogero, together with his interpretation of it, is based on the explicit consideration of the exceedingly large number of microscopic constituents forming the Universe, which may justify invoking classical chaos as the founding stone of a possible gravitational stochastic background giving origin to quantization.

In the next sections we will see that hypothesis (1) can be viewed as the consequence of a principle of classical mechanical stability, and that it leads to a characteristic unit of action per particle of the order of $h$, Planck constant, for any stable system, irrespective of the number of its constituents.

\section{GENERAL FORMULATION: THE PRINCIPLE OF STABILITY, AND THE DERIVATION OF THE COHERENT TREMOR HYPOTHESIS}

In this section we generalize the scheme of Calogero to any bound, stable classical dynamical system of global dimension $R$ and global time scale $\mathcal{T}$, formed by $N$ elementary constituents.

We will show that the characteristic action per particle for any aggregate can be connected to the related phenomenological force, and that from this procedure the tremor statement (1) follows in a natural way.

Let us consider a system described by a classical law of force $F(r)$. By introducing the mass $m$ of a generic elementary constituent, its mean global characteristic velocity $v=R / \mathcal{T}$ and its characteristic time scale $\tau$, we define the characteristic unit of action per particle as

$$
\alpha \cong m v^{2} \tau .
$$

Now, an elementary criterion of dynamical stability for classical bound systems requires that on average the characteristic potential energy of a particle must be of the same order of magnitude of its characteristic kinetic energy. As a matter of fact, this principle reduces to the virial theorem [5], complemented with the hypothesis that the mean kinetic energy per particle and the mean potential energy per particle do not depend on the particular constituent being chosen. Then, if $\mathcal{L}$ denotes the characteristic work performed on average by the system on a generic constituent, one has to impose that

$$
\mathcal{L} \cong m v^{2} .
$$

On the other hand, 


$$
\mathcal{L} \cong N F\left(R_{m}\right) R_{m} \cong N F(R) R,
$$

where $R_{m}$ denotes some mean length scale, which is obviously of the same order of magnitude of the global dimension $R$.

Eqs. (9), (10) allow to express the characteristic velocity as

$$
v \cong \sqrt{N F(R) R m^{-1}}
$$

Reminding the definition

$$
v \cong R \mathcal{T}^{-1}
$$

we can put together the definition (8) with the expressions (11), (12), to finally obtain for the characteristic unit of action per particle the following expression:

$$
\alpha \cong m \sqrt{N F(R) R m^{-1}} \frac{\tau}{\mathcal{T}}=m^{1 / 2} R^{3 / 2} \sqrt{F(R)} \frac{\tau}{\mathcal{T}} \sqrt{N} .
$$

Looking at the expression (13), we can see, now, that the fluctuation hypothesis of Calogero Eq. (1) can be immediately derived by imposing what we can denote as a "statement on the universal stability of matter", namely by requiring that the characteristic unit of action per particle be independent from the number $N$ of elementary constituents. In this way we obtain, besides the position (1) for the characteristic time $\tau$, the following form of the characteristic unit of action per particle:

$$
\alpha \cong m^{1 / 2} R^{3 / 2} \sqrt{F(R)},
$$

which connects $\alpha$ with the characteristic equilibrium dimension of the system, its characteristic constants (such as the masses of the elementary constituents) and the classical phenomenological law of force associated to it.

Application of relation (14) to specific constituents, forces, and systems will then provide the order of magnitude of the characteristic unit of action per particle $\alpha$ for the various possible physical stable aggregates.

We have thus far extended the scheme of Calogero into a more general theoretical framework. Before turning to the applications of Eq. (14) to actual physical systems in order to compute the unit of action per particle in several cases of fundamental interest, we show in the next section that the coherent tremor hypothesis (1) can be reformulated in a form indipendent by the number of constituents $N$, allowing us to introduce a characteristic scale of length $l$ connected to the characteristic time $\tau$.

\section{GENERAL FORMULATION: THE KEPLERIAN FRACTAL FORM OF THE TREMOR}

We consider at first the particular case of the Universe. In order to reformulate position (1) in a form which is independent from the number $N$ of constituents, we define the mean volume per particle $V / N$, with $V$ the mean global volume of the Universe, and a characteristic length

$$
l \cong(V / N)^{1 / 3} .
$$

The following simple chain of relations then trivially follows from Eqs. (1) and (15):

$$
\tau^{2} \cong \frac{V}{N} \frac{\mathcal{T}^{2}}{V} \cong l^{3} \frac{\mathcal{T}^{2}}{V} \cong l^{3} \frac{\mathcal{T}^{2}}{R^{3}},
$$

where we have exploited $V \cong R^{3}$, with $R$ the observed radius. On the other hand, $\mathcal{T}$ is of the same order of magnitude of the period of a particle subject to the third Kepler law and performing an orbit of radius $R$. But exactly Kepler law assures that $\mathcal{T}^{2} / R^{3}=$ const, and therefore Eq. (16) yields the relation 


$$
l \sim \tau^{2 / 3},
$$

where the symbol $\sim$ indicates proportionality. As the exponent $2 / 3$ reminds of the large scale Kepler law, we refer to relation (17) as to the "Keplerian" form of the tremor. The fractal exponent involved is a signature of the fluctuative nature of the tremor hypothesis.

Considering now stable aggregates different from the Universe, we see that, due to the simple fact that these systems must have well defined volume $V\left(\cong R^{3}\right)$ and characteristic unit of time $\mathcal{T}$, it is trivially true that $\mathcal{T}^{2} / R^{3}=$ const, independently from the validity of Kepler law. Then, the statement (1), the definition (15) and the chain of relations (16) will lead again to relation (17). Also in this case we will speak of "Keplerian" form of the tremor.

Some comments are now in order. The definition (15) allows to interpret the characteristic length $l$ as a "mean free path" per particle, but also as the "mean position uncertainty" per particle; this last interpretation could be more suitable as long as quantum aspects are involved.

As already pointed out, the term "Keplerian form" for relation (17) is not referred, in general, to some periodic orbit and a true Kepler law, but it is only reminiscent of the Kepler $2 / 3$ exponent. The interesting features of the form (17) are its independence from the number $N$ of constituing particles, and its nonanalytic character which is typical of classical stochastic fluctuations.

\section{COMPUTATION OF THE CHARACTERISTIC UNIT OF ACTION PER PARTICLE FOR GENERIC STABLE DYNAMICAL SYSTEMS}

We will now use expression (14) to compute the order of magnitude of the characteristic unit of action per particle associated to several stable aggregates of constituents interacting through different (overall attractive) laws of force.

\subsection{Gravitational interactions}

In this case, we insert in eq. (14) the gravitational law of force $F(R)=G m^{2} / R^{2}$ between pairs of nucleons (hydrogen atoms) of mass $m \cong 10^{-27} \mathrm{Kg}$, calculated at some average distance of the same order of magnitude of the global size of the system, i.e. the observed radius of the Universe $R \cong 10^{30} \mathrm{~m}$. The result is just the expression (7) for the quantum of action (Planck constant), originally derived by Calogero.

\subsection{Electromagnetic interactions}

This case requires a somewhat more accurate analysis with respect to the gravitational one. To begin with, one can consider the most elementary stable aggregate of electromagnetic nature, provided by the electrostatic interaction between two elementary charges of opposite sign, for instance an electron and a proton in a hydrogen atom: $F=e^{2} / R^{2}$ (we use electrostatic units throghout). The basic relations (13) and (14) then yield

$$
\alpha \cong m^{1 / 2} e R^{1 / 2}
$$

Here $e \cong 10^{-14} N^{1 / 2} \mathrm{~m}$ is the elementary charge, while the mean interparticle distance, of the order of magnitude of the size of the system, is of course taken to be the Bohr radius: $R \cong 10^{-10} \mathrm{~m}$; finally, for $m$ we choose the reduced mass between electron and proton masses, whose value is practically of the order of magnitude of the electron mass $m \cong 10^{-30} \mathrm{Kg}$.

Inserting these numerical values in Eq. (18) we obtain again $\alpha \cong h$, the Planck action constant.

The situation becomes even more intriguing when one considers more complex systems formed by many charged particles subject to screening effects of the charge distributions, and to continous processes of photoemission and absorption. Electromagnetic interactions exhibit a wide range of validity, which extends from the microscopic scale of the hydrogen atom $\left(\cong 10^{-10} \mathrm{~m}\right)$ to the intermediate scales typical of large molecules and molecular clusters $\left(\cong 10^{-6} \mathrm{~m}\right)$, up to the macroscopic scales. 
In the present context, one can take into account screening effects and the permanent emission and reabsorbtion of photons by including in our treatment a new fundamental constant ruling these processes. For electromagnetic interactions this cannot be else than the velocity of light $c$. The computation of the characteristic action per particle becomes then dependent on the phenomenological laws of force that can be built by taking combinations of powers not only of $e, m$, and $R$, but also of $c$. We can take the exponents of the powers of the former three quantities to be parametrically dependent on the exponent, say $\gamma$, of the powers of $c$ :

$$
F\left(e, m, R, c^{\gamma}\right)=\left(e^{2}\right)^{a(\gamma)} m^{b(\gamma)} R^{d(\gamma)} c^{\gamma},
$$

and then proceed to determine them for each chosen value of $\gamma$. The value $\gamma=0$ of course yields Coulomb law (no screening effects, neglect photoemissions and absorbtions). Negative values of $\gamma$ define screened Coulomb forces, that decay faster than $R^{-2}$. We now give some examples:

a) $\gamma=-1$ : in this case

$$
F=\frac{e^{3}}{m^{1 / 2} c R^{5 / 2}},
$$

and this leads to a unit of action per particle that has the following expression:

$$
\alpha=m^{1 / 4} e^{3 / 2} c^{-1 / 2} R^{1 / 4} .
$$

Due to the exponent $1 / 4$ the variation of $R$ over a wide range of values does not sensibly affect the order of magnitude of $\alpha \cong h$; however, the equality is optimized for $R \cong 10^{-6} \mathrm{~m}$, which corresponds to the size of large molecules and molecular clusters.

b) $\gamma=-2$ : in this case

$$
F=\frac{e^{4}}{m c^{2} R^{3}},
$$

and this yields a unit of action

$$
\alpha=\frac{e^{2}}{c} .
$$

We see that in this case the dependence of $\alpha$ on the dimensions of the system disappears. Moreover, the unit of action turns out to be the ratio $\hbar / \alpha_{e m}$, i.e. of Planck constant to the electromagnetic fine structure constant.

We have not got at present a clear understanding of this very intriguing result and of its possible deep significance. However, we notice that the effective dipole-dipole force given by eq. (22) is known to be responsible for the binding of dipolar crystals. The latter can exist in chains of more or less arbitrary length, ranging from atomic dimensions (monocellular crystals), up to macroscopic ones, and are moreover very stable. This observation in some sense justifies the absence of the length scale $R$ of the aggregate in eq. (23). The latter relation can then be interpreted as a crude approximation to a dependence given by a very small power: $\alpha \sim R^{\delta}$, with $\delta$ very close to zero. However, the appearance of the fine structure constant and its relation to the fluctuative hypothesis and to dipolar effective interactions is more intriguing and deserves to be better understood.

In conclusion, we have shown that by taking into account screening effects and photoemission in some effective way by inserting powers of $c$ in the determination of the phenomenological law of force, we can move smoothly from the microscopic domain towards larger scales and more complex structures, by requiring the unit of action per particle to be fixed at the value (in order of magnitude) of Planck constant. Viceversa, fixing the dimensions of the mesoscopic and macroscopic aggregates in the average expressions of the phenomenological force laws, and inserting the latter in our expression for the characteristic unit of action per particle, yields (in order of magnitude) Planck constant. 
However, in order to support our point of view, it is crucial to test our scheme in the cases of truly macroscopic charged aggregates, which are universally recognized, like in the case of gravitational interactions, to be included in the classical domain, and whose dynamics is commonly studied in the framework of classical mechanics. We move then to consider two relevant instances of such systems: neutral plasmas and charged beams in particle accelerators.

The case of neutral plasmas [6] is somewhat problematic. First of all, these systems are only approximately in a full equilibrium state (for instance one cannot define a global equilibrium temperature, but one can only attribute a different temperature for each kind of charged particle species that is present). Since our method is explicitely designed to deal with stable, bound systems, we cannot "a priori" expect such a spectacular appearance of the Planck constant (in order of magnitude) for the unit of action per particle in each specific instance of neutral plasmas.

In fact, we cannot even define unambigously a "global" characteristic dimension, in particular, a global coherence length. However, we can consider data connected to the plasma oscillations, which in globally neutral plasmas are described by an elastic effective force

$$
F=-k_{p} x .
$$

acting on electrons $[6]$. Here, $x$ describes the distance inside a double stratus of opposite charges, while the elastic constant $k_{p}$ is given by [6]

$$
k_{p}=4 \pi n_{e} e^{2},
$$

with $n_{e}$ the number of electrons per unit of volume and $e$ the electron charge $e \cong 10^{-14} N^{1 / 2} \mathrm{~m}$. Finally, the characteristic linear dimension is given by the Debye radius $R_{D}$, which represents the maximal elongation of the oscillations.

We are then describing the neutral plasma as composed by subsystems (the oscillating strata) which can be considered, to some extent depending on the particular plasma being considered, nearly mutually independent and in dynamical equilibrium. Obviously, this description will be valid only in some crude approximation.

We apply now relation (14) to these subsystems, choosing for the mass that of an electron, and for the characteristic dimension associated to stability the Debye radius. We obtain then

$$
\alpha \cong m^{1 / 2} k_{p}{ }^{1 / 2} R_{D}{ }^{2} .
$$

At this point we shall consider that one is concerned with a wide variety of plasma systems: celestial plasmas (ionosphere, solar photosphere, interstellar matter), gaseous discharge plasmas, thermonuclear fusion plasmas, and so on, each associated to particular values of the parameters [6].

As we have already remarked above, we cannot expect to obtain $\alpha \cong h$ in all such cases. However, for high pressure and extremely high pressure gaseous discharge plasmas (for which $n_{e}=10^{21} \mathrm{~m}^{-3}$, $R_{D}=10^{-7} \mathrm{~m}$ and $n_{e}=10^{23} \mathrm{~m}^{-3}, R_{D}=10^{-9} \mathrm{~m}$, respectively), for plasmas in stationary regimes induced by lasers (for which $n_{e}=10^{17} \mathrm{~m}^{-3}, R_{D}=10^{-9} \mathrm{~m}$ ), and for plasmas induced by lasers in thermonuclear fusion experiments (for which $n_{e}=10^{28} \mathrm{~m}^{-3}, R_{D}=5 \cdot 10^{-10} \mathrm{~m}$ ) [G] eq. (26) gives just $\alpha \cong h$ up at most one order of magnitude.

In the other cases of celestial, gaseous discharge and thermonuclear fusion plasmas, which evidently are in more unstable states with respect to the above cited plasmas, the order of magnitude of $\alpha$ is sensibly greater with respect to Planck constant (for each of these systems we have $\alpha \cong 10^{-29} \mathrm{~J} \cdot \mathrm{s}$ ). Finally, two cases (interstellar matter and solar horn plasmas ) cannot be treated at all, due to their strong instability and the consequent large uncertainties on the available experimental data.

In conclusion, taking into account the above exposed difficulties, we can assert that our method works in a not unsatisfactory way also for neutral plasmas, again showing the presence of relevant quantum signatures in macroscopic systems.

We now show that a much more stringent check for our scheme can be obtained by studying the case of charged beams in particle accelerators. These systems, in fact, are without doubt mantained, during 
very long time periods in a stable and "coherent" state of motion by the constant application of focussing external magnetic fields and by impulsive energy pumping from radiofrequency cavities.

In the reference frame comoving with the bunch of the beam, one can consider the transverse oscillations of a charged particle with respect to the ideal circular orbit. Also in this case the associated effective force is in first approximation elastic (neglecting in the magnetic field terms higher then the dipolar contributions):

$$
F=-k_{b} x,
$$

where $x$ is the transverse displacement and $k$ the effective elastic constant. From eq. (14) we have then

$$
\alpha \cong m^{1 / 2} k_{b}^{1 / 2} R^{2},
$$

with $m$ the mass of the particles (protons or electrons) and $R$ the transverse dimension of the bunch.

If we now insert in eq. (28) the most recent data from Hera proton accelerator and from the electron linear collider, we have that for the Hera proton accelerator $k_{b} \cong 10^{-12} N \cdot \mathrm{m}^{-1}, R \cong 10^{-7} \mathrm{~m}$, while for the electon linear collider the data are $k_{b} \cong 10^{-11} N \cdot \mathrm{m}^{-1}, R \cong 10^{-7} \mathrm{~m}$.

With these values, and by inserting respectively proton and electron masses, eq. (28) yields for $\alpha$ in both cases just the order of magnitude of Planck constant.

Closing this subsection, we may say that although our treatment based on effective forces is certainly qualitative, they yield for the characteristic action $\alpha$ the order of magnitude of Planck constant for electromagnetic systems on all spatial scale ranges, and for a number of charged constituents that varies from $N=2$ (hydrogen atom) to macroscopic values.

\subsection{Strong interactions}

We now move to consider hadrons having as granular constituents collections of bound quarks.

The interaction we consider is the "string law" described by the typical confining potential $V=k r$, with the string constant $k$ varying in the range $k \cong 0.1 \mathrm{GeV} \cdot \mathrm{fm}^{-1} \div 10 \mathrm{GeV} \cdot \mathrm{fm}^{-1}$ (values compatible with the experimental bounds [7]).

The quark masses vary in the range $m \cong 0.01 \mathrm{GeV} \cdot c^{-2} \div 10 \mathrm{GeV} \cdot c^{-2}$ [8], while the dimension $R$ is the range of nuclear forces, $R \cong 10^{-15} \mathrm{~m}$. The constant string force $F=k$, together with eq. (14), gives for the characteristic action

$$
\alpha \cong m^{1 / 2} R^{3 / 2} k^{1 / 2}
$$

If we insert in eq. (29) the numerical value of $R$, and some intermediate values for the mass $m$ and the string constant $k$, we obtain also in this case $\alpha \cong h$, the Planck constant. The natural objection at this point is that, as long as we consider systems of bound quarks, since these objects "live" in the deep quantum domain, getting Planck constant is a trivial matter, since such quantity is directly encoded in the characteristic physical parameters, in particular in the estimate of the string constant $k$.

We remark however that, besides the fact that the quark case supplies a necessary check of consistency for the congruence of our scheme, it is in any case worth noting the implicit possibility to construct an effective "semiclassical" fluctuative approximation also for quark systems.

The second comment is on the expression of the characteristic action $\alpha$ that can be obtained for a system of confined quarks by allowing the unit of action to depend on $c$, and therefore by taking into account, in analogy with electromagnetic systems, the virtual exchange of gluons and screening effects. Following the same line of reasoning adopted in the previous subsection, we end up then with the following expression for the unit of action per constituent:

$$
\alpha \cong\left(m c^{2}\right)^{3 / 2} c^{-1} k^{-1 / 2} R^{1 / 2} .
$$

Inserting numbers, we obtain again Planck action constant, up to at most one or two orders of magnitude. The force law associated to such expression for $\alpha$ is now modified with respect to the linear string law, 
as it acquires a form $\sim 1 / R^{2}$. However, this is not so surprising. First of all, a similar term must be added to the confining one in the phenomenological form of the interaction between quarks $[7]$ to take into account the repulsive effects at short distances. Furthermore, it is possible that also the string-like contribution is simply an effective description in a stable, confining region of, for instance, a three-body system of quarks, while its real two-body interaction might always be of the Gauss form $\sim 1 / R^{2}$.

We will not dwell deeper into this problem. However, it is relevant for our general line of reasoning that, following two different qualitative procedures, we always obtain $\alpha \cong h$.

\section{FLUCTUATIVE HYPOTHESIS AND TEMPERATURE: THE EQUIVALENT THERMAL UNIT OF ACTION}

In the picture we have outlined till now, a crucial role is assigned to the characteristic time $\tau$. It is then important to estimate its order of magnitude for different systems through some independent method.

Since our procedure is of a semiquantitative nature, an explicit expression for $\tau$ can provide consistency checks of the fundamental fluctuative hypothesis, to yield further support to its universal validity.

It is well known that the typical time scale of quantum fluctuations can be defined as the ratio between $h$ and a suitable energy describing the equilibrium state of a given system about its characteristic dimensions. This leads naturally to identify this energy with some equivalent thermal energy $k_{B} T$, with $k_{B}$ the Boltzmann constant and $T$ some equivalent temperature measured in units of the absolute Kelvin scale.

On the other hand, in our scheme such time scale coincides, at least in order of magnitude, with the fluctuative time $\tau$. Having tested that it always holds that $\alpha \cong h$, we then write

$$
\tau \cong \frac{h}{k_{B} T} .
$$

It is interesting to note that, with the above definition, we obtain from the universal tremor hypothesis eq. (1) the following suggestive definition of the equivalent temperature:

$$
T \cong \frac{h}{k_{B}} \frac{\sqrt{N}}{\mathcal{T}},
$$

which in turn leads to the following general definition of the equivalent thermal unit of action:

$$
A_{T, N} \equiv\left(k_{B} T\right) \mathcal{T} \cong h \sqrt{N} .
$$

We remark the nontrivial nature of this definition that links the thermal unit of action to the square root of the number of elementary constituents through the Planck action constant.

We can apply relations (31)-(33) to some well established phenomena, as a further test of validity of our theoretical scheme. We start by considering charged beams in particle accelerators.

Among the stable aggregates of charged particles, they play a paradigmatic role. They are very interesting from our point of view because, although they are generally described in classical terms due to their macroscopic dimensions [9], quantum aspects seem to be however relevant expecially for electron beams.

Moreover, quantum-like treatments of these systems, attempting descriptions based on some wave properties of the dynamics at a "mesoscopic" level appear very effective and in some cases also experimentally well verified [10].

In a companion paper [11] we introduce a quantum-like approach to charged beams whose basic ingredient is a diffusive kinematics ruled by the effective "mesoscopic" diffusion coefficient (33), which simulates semiclassical corrections to classical dynamics by means of classical stochastic processes. The quantum-like equations of motion in hydrodynamic form are then obtained by applying to the mean 
classical stochastic action the well established techniques of stochastic variational principles that have been extensively and successfully developed in the framework of Nelson stochastic mechanics, an independent and self-consistent reformulation of non relativistic quantum mechanics in the language of classical stochastic processes [12].

To the end of implementing this program is however crucila that we first obtain in our semiquantitative framework the correct order of magnitude of the characteristic physical quantities associated to charged beams. The typical quantity we are interested in is the thermal beam emittance $\mathcal{E}_{T}$ [9].

The thermal beam emittance is a scale of action (or equivalently of temperature, or length) which characterizes the spreading in phase space of the collective motion of a charged particle beam. It then plays the role of a diffusion coefficient in the stochastic treatment, and of a fundamental quantum of action in the quantum-like description of beams [10].

For various electron machines its value in units of the Compton length $\lambda_{c}=h / m c$ ( $m$ the electron mass), extrapolated from experiments, lies in a range $\mathcal{E}_{T} \cong 10^{6} \lambda_{c} \div 10^{9} \lambda_{c}$ [13].

Following our scheme we must assume that the natural expression for the characteristic thermal action associated to charged beams be given by eq. (33). Since in a typical bunch $N \cong 10^{11} \div 10^{12}$ [8], we obtain $\mathcal{E}_{T} \cong 10^{6} \lambda_{c} \cong 10^{-6} \mathrm{~m}$, in good agreement with the estimated phenomenological order of magnitude. Our scheme shows then in this case a good degree of consistency. Again, eq. (33) is remarkable in that it provides an intriguing relation between the (transverse) emittance of a charged beam and the number of particles in the beam. The connection with the square root of $N$ "summarizes" the complex fluctuative phenomena taking place in a bunch of interacting charged particles in accelerators. As the validity of eq. (33) would imply relevant consequences for physics of charged beams, it would be important to supply further theoretical arguments, or devise experimental tests which could confirm it.

We now move on to consider another very typical macroscopic system such as a gas constitued by $N \cong$ $10^{23} \mathrm{~mol}^{-1} \div 10^{24} \mathrm{~mol}^{-1}$ (the Avogadro number) particles. If we take $\mathcal{T} \cong 10^{-2} \mathrm{~s} \div 10^{-3} \mathrm{~s}$, corresponding to a rms velocity $v_{T} \cong 10^{2} \mathrm{~m} \cdot \mathrm{s}^{-1} \div 10^{3} \mathrm{~m} \cdot \mathrm{s}^{-1}$ (for gases at room temperature), we obtain from eq. (32) that the our equivalent fluctuative temperature takes the value $T \cong 10^{2} K \div 10^{3} \mathrm{~K}$, i.e. room temperature, as ith should be.

At the opposite end of the ladder, we pick up then a typical microscopic system such as a set of confined quarks in a nucleon, for which we can naturally assume $N \cong 1$. The typical energy scale $E$ of light quarks in a nucleon is of the order of $\Lambda_{Q C D} \cong 0.1 \mathrm{GeV}$ [8], corresponding to an equivalent temperature $T=E / k_{B} \cong 10^{12} \mathrm{~K}$. Being the system relativistic, the characteristic velocity is of the order of the velocity of light $c$. Since the scale of length is $R \cong 10^{-15} \mathrm{~m}$, we have $\mathcal{T} \cong R / c \cong 10^{-23} \mathrm{~s}$, and with these numerical values eq. (32) yields exactly the correct order of magnitude $T \cong 10^{12} \mathrm{~K}$.

Finally, let us consider a thermodynamic system that exhibits macroscopic quantum behavior. A typical example is provided by a Bose condensate. Bose-Einstein condensation has been recently experimentally observed in a gas of rubidium and sodium [14], and then later achived employing different chemical elements in several laboratories around the world. We here use eqs. (31) and (32) to estimate the characteristic rms velocity of an atom in the condensate.

The condensate reaches a linear dimension $R \cong 10^{-4} \mathrm{~m}$ at a temperature $T \cong 10^{-6} \mathrm{~K}$, and it contains $N \cong 10^{7}$ atoms. Letting $\mathcal{T} \cong R / v$, with $v$ the characteristic velocity, eq. (31) yields

$$
v \cong \frac{k_{B}}{h} \frac{T}{\sqrt{N}} R .
$$

Inserting numerical values, $v$ turns out to be of the order of $10^{-2} \mathrm{~m} \cdot \mathrm{s}^{-1}$ as experimentally observed.

As a last example, we come back to the most macroscopic system one may imagine, i.e. the whole Universe, and employ our scheme to estimate the total number of nucleons contained in it. To start with, we interpret, as usually done, the measured temperature associated to the cosmic background radiation, $T=2.7 K$, as the characteristic equivalent temperature of the Universe.

We insert in eq. (32) a characteristic global time $\mathcal{T} \cong R / v$, with $R$ the observed radius of the Universe and $v$ a characteristic velocity pertaining to microscopic constituents of the Universe. This velocity can 
be extimated, in the linear classical regime, by the equipartion theorem: $m v^{2} \cong k_{B} T$, which, together with eq. (32) provides the following expression for the total number $N$ of nucleons in the Universe:

$$
N \cong m k_{B} T R^{2} h^{-2},
$$

which, inserting the numerical values that were previously quoted for the nucleon mass, the Boltzmann constant, the cosmic backgrounnd temperature, the observed radius of the Universe and the Planck action constant, yields $N \cong 10^{78}$. This result, obtained in our crude qualitative framework, is in extraordinarily amazing agreement with the currently accepted central value $N \cong 10^{\nu}, \nu=78 \pm 8$, estimated by cosmological arguments 顿.

\section{CONCLUSIONS}

By postulating a general principle of mechanical stability for bound confined dynamical systems, we have derived the necessity of a classically chaotic fluctuative component of the classical motion, first introduced as a hypothesis by Calogero in the case of gravitational systems.

We have then developed a general semiquantitative scheme that allows to compute the order of magnitude of the characteristic action per constituent in systems ruled by generic, overall attractive law of forces, including the crucial instances of electromagnetic and strong interactions.

We have shown that in order of magnitude the characteristic action per particle is always equal to Planck constant for several generic aggregates with the dimensions of the different aggregates ranging from microscopic to macroscopic scales, going from systems of confined quarks in nucleons up to the whole numbre of gravitationally interacting nucleons in the Universe (the case first envisaged by Calogero).

We have thus shown that the original proposal by Calogero can be greatly extended and assumes a universal validity, irrespective of the interactions and of the systems being considered. In fact, from the universal validity of the analysis of Calogero, we infer that quantum mechanics and the law of force associated to a given confined system determine the dimensions of coherence of any physical system (from quarks in nucleons to the whole Universe) by imposing a classical principle of mechanical stability.

Our extension extracts from the original proposal by Calogero the very interesting and promising possibility of obtaining, for any physical system, fundamental quantum aspects at the classical level by introducing proper elementary classical fluctuations.

This procedure can turn out to be useful in general, and in particular for the study of phenomena lying at the boundary between the classical and the quantum domains [15]. In these regions it can be also interesting to explore whether, and to what extent, Nelson stochastic mechanics, i. e. a line of thought that associates classical stochastic processes to the dynamics of quantum states may acquire a more fundamental status rather than being just a mathematical reformulation of quantum mechanics in terms of classical stochastic processes [16].

It is worth noting that, among all the physical systems for which the scheme has been tested, a particularly relevant role is played by the macroscopic charged aggregates which, just as the Universe, are in fact commonly included in the classical domain, where Planck constant should not play any significant role. Viceversa, by remaining in a purely classical setting, we show that quantum mechanics leaves its significant footprint also on the classical arena in a higly nontrivial way. In our opinion, it is exactly the very crude and semiquantitative character of our analysis that makes this result expecially compelling. Our next effort will be to explicitely construct an algorithm which allows the present scheme to rest on more quantitative grounds.

Coming to terms with the conceptual issues that our analysis opens wide, the first thought must concern the physical origin of the coherent tremor, which remains an open question. One might say that our findings support an enlarged Calogerian point of view, asserting that a tiny chaotic (stochastic) component of the motion actually affects the dynamics of any classical system, with gravity playing no particular privileged role, and that this universal chaoticity is at the origin of quantization. The chaoticity would be universal, in spite of the differences between, say, the gravitational and the electromagnetic 
interactions, because of the scaling behavior of the couplings and of the spatial ranges of the different forces, whose ratios are adjusted to yield $h$ in all instances.

A more cautious and sensible stand is, to us, to regard our results as disclosing remarkable methodological perspectives, in particular the possibility to formally describe some relevant aspects of quantum mechanics in the mathematical language of classical dynamics complemented with that of classical stochastic processes. The universality of the procedure seems to rule out the (undoubtely suggestive) possibility of a privileged role of classical gravitation as the "origin" of quantum mechanics.

The indication seems rather to be that quantum mechanics (whatever the physical origin of it) and the particular law of force ruling a given physical system determine the coherence length of the latter under proper conditions of dynamical stability: remarkably, our analysis shows that this is also true for the whole Universe, when treated as an isolated dynamical system. The idea that quantum mechanics fundamentally determines the observed radius of the Universe is certainly intriguing per se. But, more than that, we stress that the connection between quantum mechanics and the dimensions of stability of physical systems on any arbitrary scale, which is due in many cases to extremely complex processes and needs to be explained by extremely sophisticated formalisms, is obtained in the present context via a very direct and intuitive line of thought.

For instance, it is very well known that in a stationary state the bound electron in the hydrogen atom does not radiate. This is the great achievement of quantum mechanics in explaining the stability of matter interacting via electromagnetic forces. One should anyway be aware of the fact that in classical mechanics stability of the hydrogen atom is not completely ruled out, once one realizes that the naive explanations of the classical instability (based essentially on the equipartion theorem) becomes higly questionable once one considers the much more sophisticated analyses on the complicated nonlinear interactions of the electron with the infinitely many modes of the classical electromagnetic field [17]. Now, once we consider the infinetely many modes of the classical electromagnetic field, or, if one wishes, the infinite fluctuating number of photons of the quantized electromagnetic field, the electron-proton system becomes a very complex nonlinear dyanmical system. We would then be led to consider the possibility of an enlarged Calogerian interpretation also for electromagnetic and strong interactions, considering even the most elementary aggregates compatible with such interactions as truly formed by an enormous amount of interacting constituents which would exhibit on the proper space-time scales some classical chaotic behavior of a possibly coherent nature.

To sum up, as already mentioned the highly remarkable fact is that the reasoning of Calogero and our analysis open the way to the definition of an algorithm which starts from the classical (phenomenological) formulation, and then allows room for relevant quantum footprints on the basis of a suitable, purely classical fluctuative hypothesis.

We expect that such a theoretical approach, when stated in a fully quantitative framework, might turn up to be a powerful computational and conceptual tool expecially when applied at the borderline between the classical and the quantum domain, and it is therefore worth of further studying and deeper understanding. 


\section{AKNWOLEDGEMENTS}

We are indebted to Francesco Guerra for the many discussions that we had with him concerning our work, and for the precious and far reaching insights he provided us in the many years of our scientific and personal acquaintance. His decisive advise and generous help have been of paramount importance to us at all stages of preparation of the present paper.

One of us (F. I.) aknowledges the Alexander von Humboldt-Stiftung for financial support, and the Quantum Theory Group of Prof. Dr. Martin Wilkens at the Fakultät für Physik der Universität Potsdam for hospitality while on leave of absence from the Dipartimento di Fisica dell'Università di Salerno.

\section{REFERENCES}

[1] S. De Martino, S. De Siena and F. Illuminati, Mod. Phys. Lett. B 12, 291 (1998).

[2] F. Calogero, Phys. Lett. A 228, 335 (1997).

[3] S. Weinberg, Gravitation and Cosmology: Principles and Applications of the General Theory of Relativity (Wiley, New York, 1972).

[4] P. J. Peebles, Principles of Physical Cosmology (Princeton University Press, Princeton, N. J., 1993).

[5] L. D. Landau and E. M. Lifshitz, Mechanics (Pergamon Press, Oxford, 1969).

[6] R. J. Goldstone and P. H. Rutherford, Introduction to Plasma Physics (Princeton University Press, Princeton, N. J., 1995).

[7] D. H. Perkins, Introduction to High Energy Physics (Addison Wesley, Menlo Park, $3^{\text {rd }}$ edition, 1993).

[8] A.A. V.V., Review of Particle Physics, Phys. Rev. D 54, 1 (1996).

[9] S. Chattopadhyay, AIP Conf. Proc. 127, 444 (1983); F. Ruggiero, A. Picasso and L. A. Radicati, Ann. Phys. (N. Y.) 197, 396 (1990).

[10] R. K. Varma, in Quantum-like Models and Coherence Effects, R. Fedele and S. Shuckla editors (World Scientific, Singapore, 1996), and references therein.

[11] N. Cufaro Petroni, S. De Martino, S. De Siena and F. Illuminati, Electronic Preprint physics/9803036 (1998), to appear in the Proceedings of the International Workshop on "Quantum Aspects of Beam Dynamics", held in Stanford, 4-9 January 1998.

[12] F. Guerra and L. M. Morato, Phys. Rev. D 27, 1774 (1983); E. Nelson, Quantum Fluctuations (Princeton University Press, Princeton, N. J., 1985); F. Guerra and R. Marra, Phys. Rev. D 29, 1647 (1984); L. M. Morato, Phys. Rev. D 31, 1982 (1985); F. Guerra, Ann. Inst. Henri Poincaré 49, 315 (1988).

[13] R. Fedele, G. Miele and L. Palumbo, Phys. Lett. A 194, 113 (1994), and references therein.

[14] M. H. Anderson et al., Science 269, 198 (1995); K. B. Davies et al., Phys. Rev. Lett. 75, 3969 (1995).

[15] D. de Falco, S. De Martino and S. De Siena, Phys. Rev. Lett. 49, 181 (1982); S. De Martino, S. De Siena, F. Illuminati and G. Vitiello, Mod. Phys. Lett. B 8, 977 (1994).

[16] F. Guerra, Phys. Rep. 77, 263 (1981); S. De Martino, S. De Siena and F. Illuminati, J. Phys. A 30,4117 (1997); F. Illuminati and L. Viola, J. Phys. A 28, 2953 (1995).

[17] G. Benettin, L. Galgani and A. Giorgilli, Nature 311, 444 (1984); L. Galgani, A. Giorgilli and F. Guerra, Phys. Lett. A 139, 221 (1989); L. Galgani, in Probabilistic Methods in Mathematical Physics, F. Guerra, M. I. Loffredo and C. Marchioro editors (World Scientific, Singapore, 1992). 